\title{
Dual Quaternion Functions and Its Applications
}

\author{
Su Jin Lim and Kwang Ho Shon \\ Department of Mathematics, Pusan National University, Busan 609-735, Republic of Korea \\ Correspondence should be addressed to Kwang Ho Shon; khshon@pusan.ac.kr
}

Received 7 June 2013; Accepted 23 July 2013

Academic Editor: Gue Lee

Copyright (C) 2013 S. J. Lim and K. H. Shon. This is an open access article distributed under the Creative Commons Attribution License, which permits unrestricted use, distribution, and reproduction in any medium, provided the original work is properly cited.

A dual quaternion is associated with two quaternions that have basis elements $e_{0}, e_{1}, e_{2}, e_{3}$, and $\varepsilon$. Dual numbers are often written in the form $z=\zeta+\varepsilon \zeta^{*}$, where $\varepsilon$ is the dual identity and has the properties $\varepsilon^{2}=0(\varepsilon \neq 0)$. We research the properties of some regular functions with values in dual quaternion and give applications of the extension problem for dual quaternion functions.

\section{Introduction}

Let $\mathscr{T}$ be the quaternion algebra constructed over a real antiEuclidean quadratic four-dimensional vector space. Brackx [1], Deavours [2], and Sudbery [3] researched properties of theories of a quaternion function. Naser [4] gave properties of hyperholomorphic functions, and Nôno $[5,6]$ gave properties of various hyperholomorphic functions. They obtained basic theorems such as Cauchy Theorem, Morera's Theorem, and Cauchy Integral Formula with respect to Clifford analysis. Also, we [7-10] have investigated certain properties of hyperholomorphic functions and some regular functions in Clifford analysis.

A dual quaternion algebra $\mathscr{D} \mathscr{H}$ is an ordered pair of quaternions and is constructed from real eight-dimensional vector spaces. A dual quaternion can be represented in the form $z=\zeta+\varepsilon \zeta^{*}$, where $\zeta$ and $\zeta^{*}$ are ordinary quaternions and $\varepsilon$ is the dual symbol. The quaternion can represent only rotation, while the dual quaternion can do both rotation and translation. So, the dual quaternion is used in applications to 3D computer graphics, robotics, and computer vision. Kenwright [11] gave characteristics of dual quaternions; Pennestrì and Stefanelli [12] researched some properties using dual, and Kula and Yayli [13] investigated dual split quaternions and screw motion in Minkowski 3-space.

Son [14-16] gave the extension problem for the solutions of partial differential equations in $\mathbf{R}^{n}$ and it is generalized for the solutions of the Riesz system. In this paper, we give some regular functions with values in dual quaternions and research the extension problem for regular functions with values in dual quaternions. Also, we give some applications for these problems.

\section{Preliminaries}

We consider associated Pauli matrices

$$
\begin{array}{ll}
e_{0}=\left(\begin{array}{cc}
1 & 0 \\
0 & 1
\end{array}\right), & e_{1}=\left(\begin{array}{cc}
i & 0 \\
0 & -i
\end{array}\right), \\
e_{2}=\left(\begin{array}{cc}
0 & 1 \\
-1 & 0
\end{array}\right), & e_{3}=\left(\begin{array}{ll}
0 & i \\
i & 0
\end{array}\right) .
\end{array}
$$

Then the associated Pauli matrices satisfy the triple rule as follows:

$$
e_{j}^{2}=-1, \quad e_{j} e_{k}+e_{k} e_{j}=-\delta_{j k} \quad(j, k=1,2,3),
$$

where $\delta_{j k}$ is Kronecker delta. And we let the dual symbol

$$
\varepsilon=\left(\begin{array}{ll}
0 & 1 \\
0 & 0
\end{array}\right)
$$

be a nonzero and satisfy $0 \varepsilon=\varepsilon 0=0,1 \varepsilon=\varepsilon 1=\varepsilon, \varepsilon^{2}=0$. The element $e_{0}$ is the identity and the element $\varepsilon$ is the dual identity of $\mathscr{T}$. 
The dual quaternion algebra $\mathscr{D} \mathscr{H}$ is a noncommutative and associative one of the quaternion algebra. Then

$$
\begin{array}{r}
\mathscr{D} \mathscr{H}:=\left\{z=\sum_{j=0}^{3}\left(e_{j} x_{j}+e_{j} x_{j}^{*} \varepsilon\right) \mid x_{j}, x_{j}^{*} \in \mathbf{R}\right. \\
(j=0,1,2,3)\} \\
=\left\{z=\zeta+\zeta^{*} \varepsilon \mid \zeta, \zeta^{*} \in \mathscr{T}\right\} \cong \mathscr{T} \times \mathscr{T},
\end{array}
$$

where $\zeta=\sum_{j=0}^{3} e_{j} x_{j}, \zeta^{*}=\sum_{j=0}^{3} e_{j} x_{j}^{*}$, and $x_{j}^{*}$ is a dual quaternion component of $x_{j}$. We can identify $\mathscr{D} \mathscr{H}$ with $\mathbf{C}^{4}$. The numbers of the skew field $\mathscr{D} \mathscr{H}$ of dual quaternions are

$$
\begin{aligned}
z & =\sum_{j=0}^{3}\left(e_{j} x_{j}+e_{j} x_{j}^{*} \varepsilon\right) \\
& =\sum_{j=0}^{3} e_{j} \xi_{j} \\
& =\left(\begin{array}{cc}
\xi_{0}+i \xi_{1} & \xi_{2}+i \xi_{3} \\
-\xi_{2}+i \xi_{3} & \xi_{0}-i \xi_{1}
\end{array}\right), \\
w & =\sum_{j=0}^{3}\left(e_{j} y_{j}+e_{j} y_{j}^{*} \varepsilon\right) \\
& =\sum_{j=0}^{3} e_{j} \eta_{j} \\
& =\left(\begin{array}{ll}
\eta_{0}+i \eta_{1} & \eta_{2}+i \eta_{3} \\
-\eta_{2}+i \eta_{3} & \eta_{0}-i \eta_{1}
\end{array}\right),
\end{aligned}
$$

where $\xi_{j}=x_{j}+\varepsilon x_{j}^{*}$ and $\eta_{j}=y_{j}+\varepsilon y_{j}^{*}(j=0,1,2,3)$. The dual quaternion conjugate $z^{*}$ of $z$ is

$$
\begin{aligned}
z^{*} & =\sum_{j=0}^{3}\left(\overline{e_{j}} x_{j}+\overline{e_{j}} x_{j}^{*} \varepsilon\right) \\
& =\sum_{j=0}^{3} \overline{e_{j}} \xi_{j} \\
& =\left(\begin{array}{ll}
\xi_{0}-i \xi_{1} & -\xi_{2}-i \xi_{3} \\
\xi_{2}-i \xi_{3} & \xi_{0}+i \xi_{1}
\end{array}\right),
\end{aligned}
$$

where $\overline{e_{j}}=-e_{j}$. The absolute value $|z|$ of $z$ and the inverse $z^{-1}$ of $z$ are, respectively,

$$
\begin{aligned}
& |z|=\sqrt{z z^{*}}=\sqrt{\sum_{j=0}^{3} \xi_{j}^{2}}, \\
& z^{-1}=\frac{z^{*}}{|z|^{2}} \quad(z \neq 0) .
\end{aligned}
$$

Let $\Omega$ be an open subset of $\mathbf{C}^{2} \times \mathbf{C}^{2}$ and let the dual quaternion function

$$
f: \Omega \longrightarrow \mathscr{D} \mathscr{H}
$$

satisfy

$$
\begin{aligned}
z \in \Omega & \\
\longmapsto & f(z) \\
= & \sum_{j=0}^{3} e_{j} f_{j}\left(\zeta, \zeta^{*}\right) \\
= & \left(\begin{array}{ll}
f_{0}\left(\zeta, \zeta^{*}\right)+i f_{1}\left(\zeta, \zeta^{*}\right) & f_{2}\left(\zeta, \zeta^{*}\right)+i f_{3}\left(\zeta, \zeta^{*}\right) \\
-f_{2}\left(\zeta, \zeta^{*}\right)+i f_{3}\left(\zeta, \zeta^{*}\right) & f_{0}\left(\zeta, \zeta^{*}\right)-i f_{1}\left(\zeta, \zeta^{*}\right)
\end{array}\right) \\
& \in \mathscr{D} \mathscr{H},
\end{aligned}
$$

where $f_{j}\left(\zeta, \zeta^{*}\right)=u_{j}\left(\zeta, \zeta^{*}\right)+\varepsilon u_{j}^{*}\left(\zeta, \zeta^{*}\right)$ and $u_{j}, u_{j}^{*}(j=0,1$, $2,3)$ are real-valued functions.

We use the following dual quaternion differential operators in $\mathscr{D} \mathscr{H}$ :

$$
\begin{aligned}
D & =\sum_{j=0}^{3} \overline{e_{j}} \frac{\partial}{\partial q_{j}} \\
& =\left(\begin{array}{cc}
\frac{\partial}{\partial q_{0}}-i \frac{\partial}{\partial q_{1}} & -\frac{\partial}{\partial q_{2}}-i \frac{\partial}{\partial q_{3}} \\
\frac{\partial}{\partial q_{2}}-i \frac{\partial}{\partial q_{3}} & \frac{\partial}{\partial q_{0}}+i \frac{\partial}{\partial q_{1}}
\end{array}\right),
\end{aligned}
$$

and the dual quaternion conjugates differential operators

$$
\begin{aligned}
D^{*} & =\sum_{j=0}^{3} e_{j} \frac{\partial}{\partial q_{j}} \\
& =\left(\begin{array}{cc}
\frac{\partial}{\partial q_{0}}+i \frac{\partial}{\partial q_{1}} & \frac{\partial}{\partial q_{2}}+i \frac{\partial}{\partial q_{3}} \\
-\frac{\partial}{\partial q_{2}}+i \frac{\partial}{\partial q_{3}} & \frac{\partial}{\partial q_{0}}-i \frac{\partial}{\partial q_{1}}
\end{array}\right),
\end{aligned}
$$

where $\partial / \partial q_{j}=\partial / \partial x_{j}+\varepsilon\left(\partial / \partial x_{j}^{*}\right)(j=0,1,2,3)$ and $q_{j}=$ $x_{j}+(1 / \varepsilon) x_{j}^{*}$. Then we have

$$
D D^{*}=\sum_{j=0}^{3} \frac{\partial^{2}}{\partial q_{j}^{2}}=\Delta_{q} .
$$

Definition 1. Let $\Omega$ be an open set in $\mathbf{C}^{2} \times \mathbf{C}^{2}$. A function $f(z)$ is said to be $\varepsilon$-regular in $\Omega$ if the following two conditions are satisfied:

(a) $f_{j}(j=0,1,2,3)$ are continuously differential functions in $\Omega$,

(b) $D^{*} f(z)=0$ in $\Omega$. 
Definition 2. Let $\Omega$ be an open set in $\mathbf{C}^{2} \times \mathbf{C}^{2}$. A function $f(z)$ is said to be $\varepsilon$-biregular in $\Omega$ if the following two conditions are satisfied:

(a) $f_{j}(j=0,1,2,3)$ are continuously differential functions in $\Omega$,

(b) $D^{*} f(z)=0$ and $f(z) D^{*}=0$ in $\Omega$.

The operators act for a function $f(z)$ on $\mathscr{D} \mathscr{H}$ as follows:

$$
\begin{aligned}
D^{*} f(z) & =\left(\sum_{j=0}^{3} e_{j} \frac{\partial}{\partial q_{j}}\right)\left(\sum_{j=0}^{3} e_{j} f_{j}\right) \\
& =\left(\begin{array}{ll}
D_{1}^{*} & D_{2}^{*} \\
D_{3}^{*} & D_{4}^{*}
\end{array}\right),
\end{aligned}
$$

where

$$
\begin{aligned}
D_{1}^{*}= & \left(\frac{\partial f_{0}}{\partial q_{0}}-\frac{\partial f_{1}}{\partial q_{1}}-\frac{\partial f_{2}}{\partial q_{2}}-\frac{\partial f_{3}}{\partial q_{3}}\right) \\
& +i\left(\frac{\partial f_{1}}{\partial q_{0}}+\frac{\partial f_{0}}{\partial q_{1}}+\frac{\partial f_{3}}{\partial q_{2}}-\frac{\partial f_{2}}{\partial q_{3}}\right) \\
D_{2}^{*}= & \left(\frac{\partial f_{2}}{\partial q_{0}}-\frac{\partial f_{3}}{\partial q_{1}}+\frac{\partial f_{0}}{\partial q_{2}}+\frac{\partial f_{1}}{\partial q_{3}}\right) \\
& +i\left(\frac{\partial f_{3}}{\partial q_{0}}+\frac{\partial f_{2}}{\partial q_{1}}-\frac{\partial f_{1}}{\partial q_{2}}+\frac{\partial f_{0}}{\partial q_{3}}\right) \\
D_{3}^{*}= & \left(-\frac{\partial f_{2}}{\partial q_{0}}+\frac{\partial f_{3}}{\partial q_{1}}-\frac{\partial f_{0}}{\partial q_{2}}-\frac{\partial f_{1}}{\partial q_{3}}\right) \\
& +i\left(\frac{\partial f_{3}}{\partial q_{0}}+\frac{\partial f_{2}}{\partial q_{1}}-\frac{\partial f_{1}}{\partial q_{2}}+\frac{\partial f_{0}}{\partial q_{3}}\right) \\
D_{4}^{*}= & \left(\frac{\partial f_{0}}{\partial q_{0}}-\frac{\partial f_{1}}{\partial q_{1}}-\frac{\partial f_{2}}{\partial q_{2}}-\frac{\partial f_{3}}{\partial q_{3}}\right) \\
& +i\left(-\frac{\partial f_{1}}{\partial q_{0}}-\frac{\partial f_{0}}{\partial q_{1}}-\frac{\partial f_{3}}{\partial q_{2}}+\frac{\partial f_{2}}{\partial q_{3}}\right) \\
f(z) D^{*}= & \left(\sum_{j=0}^{3} e_{j} f_{j}\right)\left(\sum_{j=0}^{3} e_{j} \frac{\partial}{\partial q_{j}}\right) \\
= & \left(\begin{array}{l}
D_{5}^{*} D_{6}^{*} \\
D_{7}^{*}
\end{array}\right),
\end{aligned}
$$

where

$$
\begin{aligned}
D_{5}^{*}= & \left(\frac{\partial f_{0}}{\partial q_{0}}-\frac{\partial f_{1}}{\partial q_{1}}-\frac{\partial f_{2}}{\partial q_{2}}-\frac{\partial f_{3}}{\partial q_{3}}\right) \\
& +i\left(\frac{\partial f_{1}}{\partial q_{0}}+\frac{\partial f_{0}}{\partial q_{1}}-\frac{\partial f_{3}}{\partial q_{2}}+\frac{\partial f_{2}}{\partial q_{3}}\right), \\
D_{6}^{*}= & \left(\frac{\partial f_{2}}{\partial q_{0}}+\frac{\partial f_{3}}{\partial q_{1}}+\frac{\partial f_{0}}{\partial q_{2}}-\frac{\partial f_{1}}{\partial q_{3}}\right) \\
& +i\left(\frac{\partial f_{3}}{\partial q_{0}}-\frac{\partial f_{2}}{\partial q_{1}}+\frac{\partial f_{1}}{\partial q_{2}}+\frac{\partial f_{0}}{\partial q_{3}}\right),
\end{aligned}
$$

$$
\begin{aligned}
D_{7}^{*}= & \left(-\frac{\partial f_{2}}{\partial q_{0}}-\frac{\partial f_{3}}{\partial q_{1}}-\frac{\partial f_{0}}{\partial q_{2}}+\frac{\partial f_{1}}{\partial q_{3}}\right) \\
& +i\left(\frac{\partial f_{3}}{\partial q_{0}}-\frac{\partial f_{2}}{\partial q_{1}}+\frac{\partial f_{1}}{\partial q_{2}}+\frac{\partial f_{0}}{\partial q_{3}}\right), \\
D_{8}^{*}= & \left(\frac{\partial f_{0}}{\partial q_{0}}-\frac{\partial f_{1}}{\partial q_{1}}-\frac{\partial f_{2}}{\partial q_{2}}-\frac{\partial f_{3}}{\partial q_{3}}\right) \\
& +i\left(-\frac{\partial f_{1}}{\partial q_{0}}-\frac{\partial f_{0}}{\partial q_{1}}+\frac{\partial f_{3}}{\partial q_{2}}-\frac{\partial f_{2}}{\partial q_{3}}\right) .
\end{aligned}
$$

Remark 3. Equations (b) of Definition 2 are equivalent to the following system:

$$
\begin{array}{ll}
\frac{\partial f_{0}}{\partial q_{0}}-\frac{\partial f_{1}}{\partial q_{1}}-\frac{\partial f_{2}}{\partial q_{2}}-\frac{\partial f_{3}}{\partial q_{3}}=0, \\
\frac{\partial f_{1}}{\partial q_{0}}=-\frac{\partial f_{0}}{\partial q_{1}}, & \frac{\partial f_{3}}{\partial q_{2}}=\frac{\partial f_{2}}{\partial q_{3}}, \\
\frac{\partial f_{2}}{\partial q_{0}}=-\frac{\partial f_{0}}{\partial q_{2}}, & \frac{\partial f_{3}}{\partial q_{1}}=\frac{\partial f_{1}}{\partial q_{3}}, \\
\frac{\partial f_{3}}{\partial q_{0}}=-\frac{\partial f_{0}}{\partial q_{3}}, & \frac{\partial f_{2}}{\partial q_{1}}=\frac{\partial f_{1}}{\partial q_{2}},
\end{array}
$$

\section{Extension Problem for the Dual Quaternion Functions}

Definition 4. Let $\Omega$ be a domain in $\mathbf{C}^{n} \times \mathbf{C}^{n}(n \geq 1)$. A function $f(z)=\sum_{j=0}^{n-1} e_{j} f_{j}(z)$ is said to be regular in $\Omega$ if

$$
\widetilde{D} f(z)=0,
$$

where $\widetilde{D}=\sum_{j=0}^{n-1} e_{j}\left(\partial / \partial q_{j}\right)$ on $\Omega$.

Theorem 5 (uniqueness theorem for regular functions). If two regular functions $f$ and $g$ in a domain $\Omega \subset \mathbf{C}^{n} \times \mathbf{C}^{n}(n \geq$ 1 ) and coincide on a nonempty open set $G \subset \Omega$, then $f \equiv g$ in $\Omega$.

Rocha-Chávez et al. [17] obtained the following remark.

Remark 6. For a regular function $f$ in the domain $\Omega \subset \mathbf{C}^{n} \times$ $\mathrm{C}^{n}(n \geq 1)$ and a bounded domain $G$ with smooth boundary $b G$, such that $\bar{G} \subset \Omega$, one has

$$
f(z)=\frac{1}{a_{n}} \int_{b G} \frac{\bar{\zeta}-\bar{z}}{|\zeta-z|^{n}} d \sigma_{\zeta} f(\zeta), \quad z \in G,
$$

with $a_{n}$ the area of the unit sphere in $\mathbf{C}^{n}$ and $d \sigma_{\zeta}$ a Clifford algebra valued differential form of order $n-1$.

Let $\Omega=\Omega_{1} \times \Omega_{2}$ be a domain in $\mathbf{C}^{4} \times \mathbf{C}^{n-4}(n \geq$ 5) where $\Omega_{1}$ is a domain in $\mathbf{C}^{4}\left(\xi_{0}, \xi_{1}, \xi_{2}, \xi_{3}\right)$ and $\Omega_{2}$ is a domain in $\mathbf{C}^{n-4}\left(\xi_{4}, \xi_{5}, \ldots, \xi_{n-1}\right)$. Let $U$ be an open connected neighborhood of $b \Omega$. 
Proposition 7. If $f(z)$ is a regular function in $U \subset \mathbf{C}^{4} \times$ $\mathbf{C}^{n-4}(n \geq 5)$ which satisfies the condition

$$
D^{*} f(z)=0
$$

then $f(z)$ can be extended continuously to a regular function in the whole domain of $\Omega$. That is, there exists a regular function $\widetilde{f}(z)$ in $\Omega$ such that $\widetilde{f}(z)=f(z)$ in $U$.

Proof. By Remark 6 and the proof of the main extension theorem of Son [15], it is proved.

We consider the system of an extension of the system (16)

$$
\begin{array}{r}
\frac{\partial f_{0}}{\partial q_{0}}-\frac{\partial f_{1}}{\partial q_{1}}-\cdots-\frac{\partial f_{n-1}}{\partial q_{n-1}}=0, \\
\frac{\partial f_{j}}{\partial q_{0}}=-\frac{\partial f_{0}}{\partial q_{j}}, \quad \frac{\partial f_{l}}{\partial q_{k}}=\frac{\partial f_{k}}{\partial q_{l}} \\
(j, k, l=1, \ldots, n-1),
\end{array}
$$

where $f(z)=\left\{f_{0}(z), f_{1}(z), \ldots, f_{n-1}(z)\right\}$ are the unknown functions.

By using the same technique as in Son [15], we have the following theorem.

Theorem 8. Let $f(z)=\left\{f_{0}(z), f_{1}(z), \ldots, f_{n-1}(z)\right\}$ be a given $\mathscr{C}^{2}$-solution of the system (20) in $U \subset \mathbf{C}^{4} \times \mathbf{C}^{n-4}(n \geq 5)$, which satisfies the system (16) in Remark 3. If the functions $f_{4}(z), f_{5}(z), \ldots, f_{n-1}(z)$ depend only on $\xi_{0}, \xi_{1}, \xi_{2}, \xi_{3}$, and $U$ is an open neighborhood of the boundary of the domain $\Omega \subset$ $\mathbf{C}^{4} \times \mathbf{C}^{n-4}(n \geq 5)$, then $f(z)$ can be extended to a solution of the system (20) in the whole domain of $\Omega$.

Proof. Let the function $\phi(z)$ with values in Clifford algebra be defined by

$$
\phi(z)=\sum_{j=0}^{n-1} f_{j} e_{j}
$$

Then we have $\widetilde{D} \phi(z)=0$ and $D^{*} \phi(z)=0$. By Proposition 7 , the result follows.

We consider the following system:

$$
\sum_{j=0}^{n-1} \sum_{k=0}^{m-1} H_{j k}^{(P)}(z) \frac{\partial f_{j}}{\partial q_{k}}=0 \quad(P=1, \ldots, p),
$$

where $H_{j k}^{(P)}(z)$ are holomorphic functions and $f=\left\{f_{0}, f_{1}\right.$, $\left.\ldots, f_{n-1}\right\}$ are the unknown functions of the system (22).

Let $\Omega$ be an open set in $\mathbf{C}^{n}(n \geq 2)$ and let $K$ be a compact subset of $\Omega$ such that $\Omega \backslash K$ is simply connected. We consider the system

$$
\sum_{j=0}^{n-1} \sum_{k=0}^{m-1} H_{j k}^{(P)}(z) \frac{\partial \omega_{j}}{\partial q_{k}}=\varphi_{(z)}^{(P)} \quad(P=1, \ldots, p),
$$

where $\varphi_{(z)}^{(P)} \in \mathscr{C}^{\infty}(\Omega)$.

By using the same technique as in Son [16], we have the following theorem and example.
Theorem 9. If every $\varphi=\left\{\varphi^{(1)}, \ldots, \varphi^{(p)}\right\} \in \mathscr{C}^{\infty}(\Omega)$ in the inhomogeneous system (23) has a solution

$$
\omega=\left\{\omega_{0}, \ldots, \omega_{n-1}\right\} \in \mathscr{C}^{\infty}(\Omega),
$$

then every solution $f$ of (22) given in $\Omega \backslash K$ can be extended to a solution of this system (23) in the whole domain of $\Omega$.

Proof. This result follows from the theorem in [18, page 30].

Example 10. We give an application of Theorem 9 to the system (20) and recall the system (16) as follows:

$$
\begin{array}{r}
\frac{\partial f_{0}}{\partial q_{0}}-\frac{\partial f_{1}}{\partial q_{1}}-\cdots-\frac{\partial f_{n-1}}{\partial q_{n-1}}=0, \\
\frac{\partial f_{j}}{\partial q_{0}}+\frac{\partial f_{0}}{\partial q_{j}}=0, \quad \frac{\partial f_{l}}{\partial q_{k}}-\frac{\partial f_{k}}{\partial q_{l}}=0 \\
(j, k, l=1, \ldots, n-1) .
\end{array}
$$

Assume that

$$
\frac{\partial f_{n-1}}{\partial q_{n-1}}=0
$$

Then we have the following form:

$$
\begin{array}{r}
\frac{\partial f_{0}}{\partial q_{0}}-\frac{\partial f_{1}}{\partial q_{1}}-\cdots-\frac{\partial f_{n-2}}{\partial q_{n-2}}=0, \\
\frac{\partial f_{j}}{\partial q_{0}}+\frac{\partial f_{0}}{\partial q_{j}}=0, \quad \frac{\partial f_{l}}{\partial q_{k}}-\frac{\partial f_{k}}{\partial q_{l}}=0 \\
(j, k, l=1, \ldots, n-2) .
\end{array}
$$

The corresponding inhomogeneous system of (27) has the following form:

$$
\begin{aligned}
& \frac{\partial \omega_{0}}{\partial q_{0}}-\frac{\partial \omega_{1}}{\partial q_{1}}-\cdots-\frac{\partial \omega_{n-2}}{\partial q_{n-2}}=\varphi, \\
& \frac{\partial \omega_{j}}{\partial q_{0}}+\frac{\partial \omega_{0}}{\partial q_{j}}=\varphi_{j, 0}, \\
& \frac{\partial \omega_{j}}{\partial q_{k}}-\frac{\partial \omega_{k}}{\partial q_{j}}=\varphi_{j, k}, \\
& \frac{\partial \omega_{k}}{\partial q_{0}}+\frac{\partial \omega_{0}}{\partial q_{k}}=\varphi_{k, 0}, \\
& \frac{\partial \omega_{j}}{\partial q_{l}}-\frac{\partial \omega_{l}}{\partial q_{j}}=\varphi_{j, l}, \\
& \frac{\partial \omega_{k}}{\partial q_{l}}-\frac{\partial \omega_{l}}{\partial q_{k}}=\varphi_{k, l} \\
& \quad(j, k, l=1, \ldots, n-2),
\end{aligned}
$$


where $\varphi, \varphi_{j, 0}, \varphi_{j, k}, \varphi_{k, 0}, \varphi_{j, l}, \varphi_{k, l} \in \mathscr{C}^{\infty}(\Omega)$. Then we can get the system from (28) as

$$
\begin{gathered}
\frac{\partial \omega_{k}}{\partial q_{j}}=\frac{\partial \omega_{j}}{\partial q_{k}}-\varphi_{j, k}, \quad \frac{\partial \omega_{k}}{\partial q_{0}}=-\frac{\partial \omega_{0}}{\partial q_{k}}+\varphi_{k, 0}, \\
\frac{\partial \omega_{l}}{\partial q_{j}}=\frac{\partial \omega_{j}}{\partial q_{l}}-\varphi_{j, l}, \quad \frac{\partial \omega_{l}}{\partial q_{k}}=\frac{\partial \omega_{k}}{\partial q_{l}}-\varphi_{k, l}, \\
\frac{\partial^{2} \omega_{k}}{\partial q_{j} \partial q_{0}}+\frac{\partial \varphi_{j, k}}{\partial q_{0}}=-\frac{\partial^{2} \omega_{0}}{\partial q_{j} \partial q_{k}}+\frac{\partial \varphi_{j, 0}}{\partial q_{k}} \\
\frac{\partial^{2} \omega_{j}}{\partial q_{l} \partial q_{k}}-\frac{\partial \varphi_{j, l}}{\partial q_{k}}=\frac{\partial^{2} \omega_{k}}{\partial q_{j} \partial q_{l}}-\frac{\partial \varphi_{k, l}}{\partial q_{j}} .
\end{gathered}
$$

From (29), we have

$$
\begin{gathered}
\frac{\partial}{\partial q_{j}}\left(-\frac{\partial \omega_{0}}{\partial q_{k}}+\varphi_{k, 0}\right)+\frac{\partial \varphi_{j, k}}{\partial q_{0}}=-\frac{\partial^{2} \omega_{0}}{\partial q_{j} \partial q_{k}}+\frac{\partial \varphi_{j, 0}}{\partial q_{k}}, \\
\frac{\partial}{\partial q_{l}}\left(\frac{\partial \omega_{k}}{\partial q_{j}}+\varphi_{j, k}\right)-\frac{\partial \varphi_{j, l}}{\partial q_{k}}=\frac{\partial^{2} \omega_{k}}{\partial q_{j} \partial q_{l}}-\frac{\partial \varphi_{k, l}}{\partial q_{j}} .
\end{gathered}
$$

Thus, we can have the system

$$
\frac{\partial \varphi_{k, 0}}{\partial q_{j}}+\frac{\partial \varphi_{j, k}}{\partial q_{0}}=\frac{\partial \varphi_{j, 0}}{\partial q_{k}}, \quad \frac{\partial \varphi_{j, k}}{\partial q_{l}}-\frac{\partial \varphi_{j, l}}{\partial q_{k}}=-\frac{\partial \varphi_{k, l}}{\partial q_{j}} .
$$

We let

$$
\widetilde{\omega}=\sum_{j=0}^{n-2} e_{j} \omega_{j}, \quad \widetilde{z}=\sum_{j=0}^{n-2} \overline{e_{j}} \xi_{j} .
$$

The system (29) has the form

$$
\begin{array}{ll}
\widetilde{D} \widetilde{\omega}=F\left(\widetilde{z}, \xi_{n-1}\right), & \frac{\partial \omega_{0}}{\partial q_{k}}+\frac{\partial \omega_{k}}{\partial q_{0}}=\varphi_{k, 0} \\
\frac{\partial \omega_{j}}{\partial q_{l}}-\frac{\partial \omega_{l}}{\partial q_{j}}=\varphi_{j, l}, & \frac{\partial \omega_{k}}{\partial q_{l}}-\frac{\partial \omega_{l}}{\partial q_{k}}=\varphi_{k, l}
\end{array}
$$

where

$$
\begin{aligned}
F\left(\widetilde{z}, \xi_{n-1}\right)= & \varphi+\sum_{j=1}^{n-2} e_{j} \varphi_{j, 0} \\
& +\left(\frac{n}{2}-1\right) \sum_{\alpha=1}^{n-2} \sum_{\substack{j, k=1 \\
j, k \neq \alpha}}^{n-2} e_{\alpha} \varphi_{j, k} .
\end{aligned}
$$

We put

$$
\begin{gathered}
F_{1}:=\varphi+\sum_{j=1}^{n-2} e_{j} \varphi_{j, 0}, \\
F_{2}:=\left(\frac{n}{2}-1\right) \sum_{\substack{\alpha=1 \\
j-2}}^{n-2} \sum_{\substack{j, k=1 \\
j, k \neq \alpha}}^{n-2} e_{\alpha, k} .
\end{gathered}
$$

From the system (28), we have

$$
\begin{gathered}
\omega_{0}=\int\left(\varphi+\frac{\partial \omega_{1}}{\partial q_{1}}+\frac{\partial \omega_{2}}{\partial q_{2}}+\cdots+\frac{\partial \omega_{n-2}}{\partial q_{n-2}}\right) d q_{0}, \\
\omega_{k}=\int\left(\varphi_{k, 0}-\frac{\partial \omega_{0}}{\partial q_{k}}\right) d q_{0} \quad(k=1, \ldots, n-2) .
\end{gathered}
$$

By the systems (29) and (38), we get

$$
\begin{aligned}
\widetilde{\omega}= & \int F d q_{0}+\int \sum_{j=1}^{n-2} \frac{\partial \omega_{j}}{\partial q_{j}} d q_{0} \\
& -\int \sum_{j=1}^{n-2} e_{j} \frac{\partial \omega_{0}}{\partial q_{j}} d q_{0}-\int F_{2} d q_{0} \\
= & \int F d q_{0}-\int \sum_{j=1}^{n-2} e_{j} \frac{\partial \widetilde{\omega}}{\partial q_{j}} d q_{0} .
\end{aligned}
$$

By Cauchy Integral Formula,

$$
F\left(Z, \xi_{n-1}\right)=\frac{1}{(2 \pi i)^{n}} \int \frac{F\left(Z, \xi_{n-1}\right)}{Z-\zeta} d Z,
$$

where $Z=\sum_{k=0}^{n-2} e_{k} Z_{k}$. Thus we have

$$
\widetilde{\omega}=\frac{1}{(2 \pi i)^{n}} \iint \frac{F\left(Z, \xi_{n-1}\right)}{Z-\zeta} d Z_{0} d Z,
$$

and $\widetilde{\omega}=0$ when $\left|\xi_{n-1}\right|$ is large enough. Also, $\{\widetilde{\omega}, 0\}=$ $\left\{\omega_{0}, \omega_{1}, \ldots, \omega_{n-2}, 0\right\}$ is a solution of the system (22) outside a compact set $K$ of $\Omega$. From Theorem 5 , it follows that $\widetilde{\omega}=0$ is outside the compact set $K$ of $\Omega$ or $\omega \in \mathscr{C}^{\infty}(\Omega)$. That is, $\omega_{0}, \omega_{1}, \ldots, \omega_{n-2} \in \mathscr{C}^{\infty}(\Omega)$. It follows from the system (35) that

$$
\omega_{n-1}=\int\left(\frac{\partial \omega_{m-1}}{\partial q_{n-1}}-\varphi_{m-1, n-1}\right) d q_{m-1} .
$$

Since $\omega_{m-1} \in \mathscr{C}^{\infty}(\Omega)$, we get $\partial \omega_{m-1} / \partial q_{n-1} \in \mathscr{C}^{\infty}(\Omega)$. We can choose $\omega_{n-1} \in \mathscr{C}^{\infty}(\Omega)$ which satisfy the system (35). From (42), we find that

$$
\begin{aligned}
& \frac{\partial \omega_{n-1}}{\partial q_{P}} \\
& =\int\left(\frac{\partial^{2} \omega_{m-1}}{\partial q_{P} \partial q_{n-1}}-\frac{\partial \varphi_{m-1, n-1}}{\partial q_{P}}\right) d q_{m-1} \\
& =\int\left(\frac{\partial}{\partial q_{n-1}}\left(\frac{\partial \omega_{P}}{\partial q_{m-1}}\right)+\frac{\partial \varphi_{m-1, P}}{\partial q_{n-1}}-\frac{\partial \varphi_{m-1, n-1}}{\partial q_{P}}\right) d q_{m-1} \\
& =\int\left(\frac{\partial}{\partial q_{n-1}}\left(\frac{\partial \omega_{P}}{\partial q_{m-1}}\right)-\frac{\partial \varphi_{P, n-1}}{\partial q_{m-1}}\right) d q_{m-1} \\
& =\frac{\partial \omega_{P}}{\partial q_{n-1}}-\varphi_{P, n-1} .
\end{aligned}
$$

Hence, $\omega_{n-1}$ satisfies the system (29). This means that the function $\omega=\left(\widetilde{\omega}, \omega_{n-1}\right)=\left(\omega_{0}, \ldots, \omega_{n-1}\right)$ is a solution of the system (29) and $\omega \in \mathscr{C}^{\infty}(\Omega)$. 


\section{Acknowledgment}

The second author was supported by Basic Science Research Program through the National Research Foundation of Korea (NRF) funded by the Ministry of Science, ICT and Future Planning (2013R1A1A2008978).

\section{References}

[1] F. Brackx, "On (k)-monogenic functions of a quaternion variable," in Function Theoretic Methods in Differential Equations, vol. 8 of Research Notes in Mathematics, pp. 22-44, 1876.

[2] C. A. Deavours, "The quaternion calculus," The American Mathematical Monthly, vol. 80, pp. 995-1008, 1973.

[3] A. Sudbery, "Quaternionic analysis," Mathematical Proceedings of the Cambridge Philosophical Society, vol. 85, no. 2, pp. 199224, 1979.

[4] M. Naser, "Hyperholomorphic functions," Silberian Mathematical Journal, vol. 12, pp. 959-968, 1971.

[5] K. Nôno, "Hyperholomorphic functions of a quaternion variable," Bulletin of Fukuoka University of Education, vol. 32, pp. 21-37, 1982.

[6] K. Nôno, "Domains of Hyperholomorphic in $C^{2} \times C^{2}$," Bulletin of Fukuoka University of Education, vol. 36, pp. 1-9, 1987.

[7] J. Kajiwara, X. D. Li, and K. H. Shon, "Function spaces in complex and Clifford analysis, inhomogeneous caychy riemann system of quaternion and Clifford analysis in ellipsoid," in Proceedings the 14th International Conferences on Finite or Infinite Dimensional Complex Analysis and Applications, vol. 14, pp. 127-155, Hue University, Hue, Vietnam, 2006.

[8] S. J. Lim and K. H. Shon, "Properties of hyperholomorphic functions in Clifford analysis," East Asian Mathematical Journal, vol. 28, pp. 553-559, 2012.

[9] S. J. Lim and K. H. Shon, "Hyperholomorphic functions and hyper-conjugate harmonic functions of octonion variables," Journal of Inequalities and Applications, vol. 77, pp. 1-8, 2013.

[10] S. J. Lim and K. H. Shon, "Regularities of functions with values in $\mathrm{C}(n)$ of matrix algebras $M(n ; \mathrm{C})$," Journal of Inequalities and Applications. Preprint.

[11] B. Kenwright, "A beginners guide to dual-quaternions: what they are, how they work, and how to use them for 3D character hierarchies," in Proceedings of the 20th International Conferences on Computer Graphics, Visualization and Computer Vision, pp. 1-10, 2012.

[12] E. Pennestrì and R. Stefanelli, "Linear algebra and numerical algorithms using dual numbers," Multibody System Dynamics, vol. 18, no. 3, pp. 323-344, 2007.

[13] L. Kula and Y. Yayli, "Dual split quaternions and screw motion in Minkowski 3-space," Iranian Journal of Science and Technology A, vol. 30, no. 3, pp. 245-258, 2006.

[14] Lê Hùng Son, "An extension problem for solutions of partial differential equations in $\mathbb{R}^{n}$," Complex Variables, vol. 15 , no. 2 , pp. 87-92, 1990.

[15] L.H. Son, "Extension problem for functions with values in a Clifford algebra," Archiv der Mathematik, vol. 55, no. 2, pp. 146$150,1990$.

[16] L. H. Son, "Extension problem for the solutions of partial differential equations in $\mathbb{R}^{n}$," Complex Variables, vol. 18, no. 12, pp. 135-139, 1992.

[17] R. Rocha-Chávez, M. Shapiro, and F. Sommen, Integral theorems for functions and differential forms in $C^{m}$, vol. 428 of
Research Notes in Mathematics, Chapman \& Hall/CRC, Boca Raton, Fla, USA, 2002.

[18] L. Hörmander, An Introduction to Complex Analysis in Several Variables, North-Holland, Amsterdam, The Netherlands, 1966. 


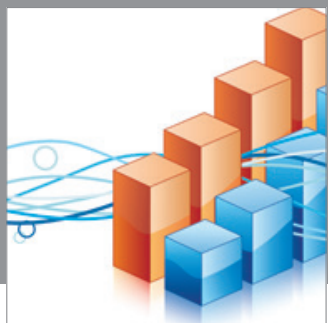

Advances in

Operations Research

mansans

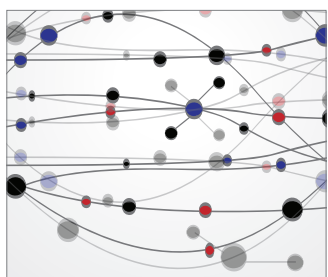

The Scientific World Journal
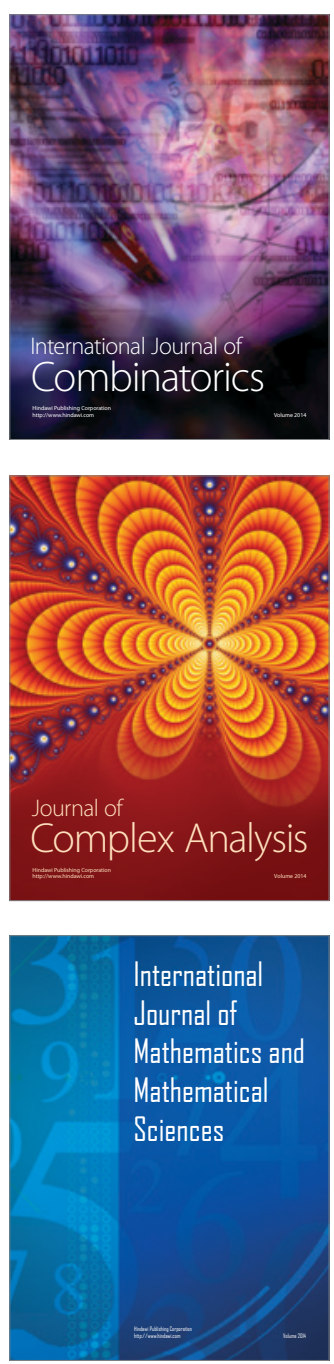
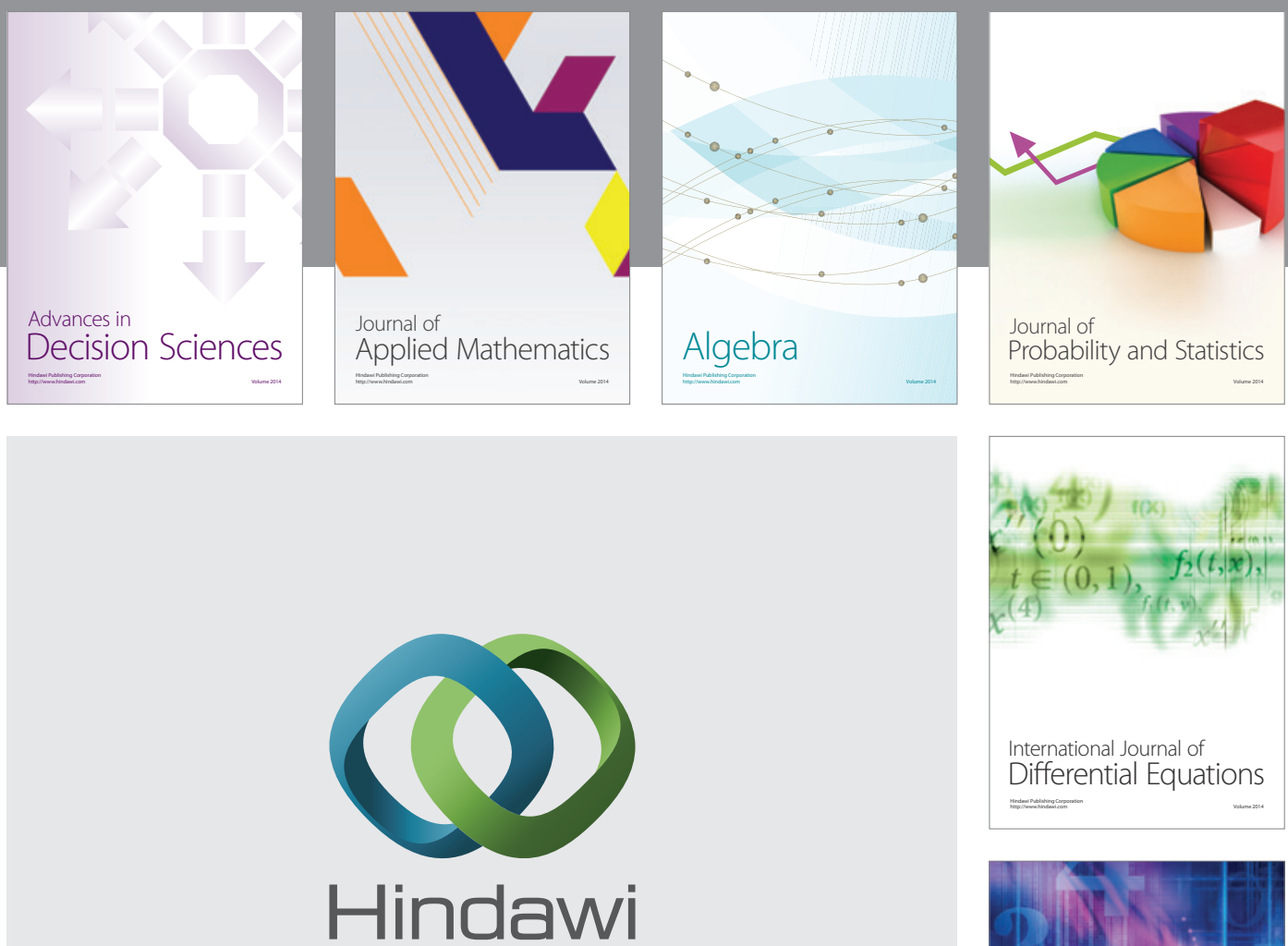

Submit your manuscripts at http://www.hindawi.com
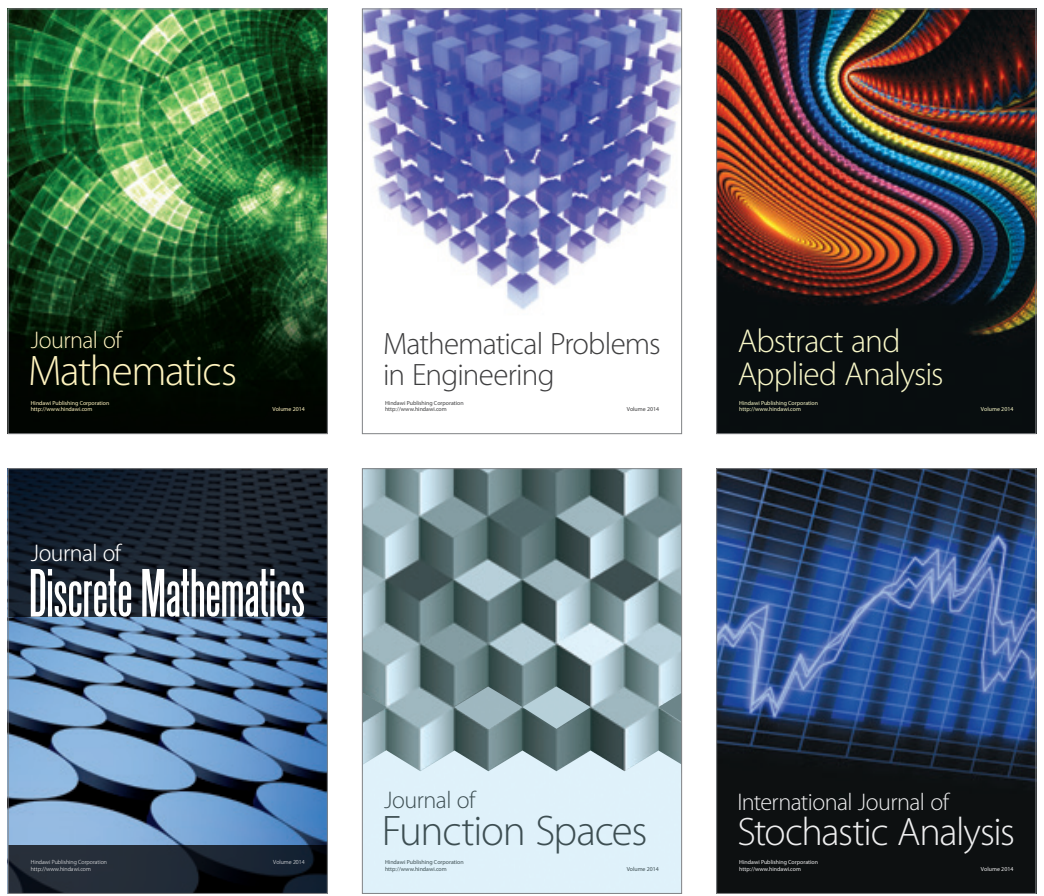

Journal of

Function Spaces

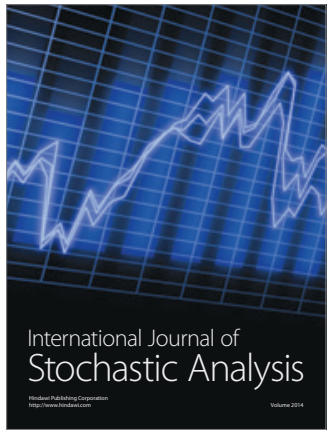

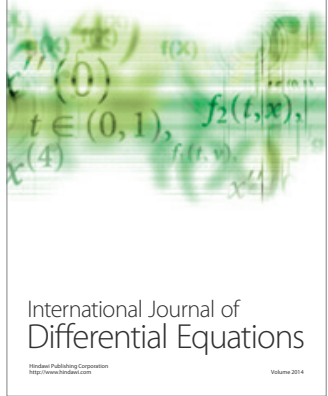
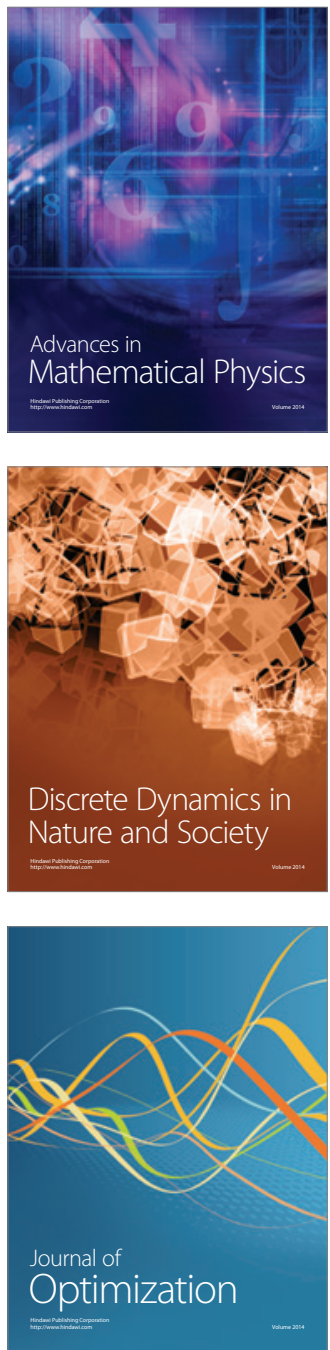